STRUCTURE REPORTS

ISSN $1600-5368$

Received 23 October 2014

Accepted 20 November 2014

Edited by M. Weil, Vienna University of Technology, Austria

Keywords: crystal structure; hexanuclear compounds; lanthanide compound; threedimensional hydrogen-bonded network

CCDC reference: 1035218

Supporting information: this article has supporting information at journals.iucr.org/e

\section{Crystal structure of $\left[\mathrm{Y}_{6}\left(\mu_{6}-\mathrm{O}\right)\left(\mu_{3}-\mathrm{OH}\right)_{8}\left(\mathrm{H}_{2} \mathrm{O}\right)_{24}\right] \mathrm{I}_{8} \cdot-$ $8 \mathrm{H}_{2} \mathrm{O}$}

François Le Natur, Guillaume Calvez, Olivier Guillou,* Carole Daiguebonne and Kevin Bernot

INSA, UMR 6226, Institut des Sciences Chimiques de Rennes, 35708 Rennes, France. *Correspondence e-mail: olivier.guillou@insa-rennes.fr

The crystal structure of the title compound \{systematic name: octa- $\mu_{3^{-}}$ hydroxido- $\mu_{6}$-oxido-hexakis[tetraaquayttrium(III)] octaiodide octahydrate\}, is characterized by the presence of the centrosymmetric molecular entity $\left[\mathrm{Y}_{6}\left(\mu_{6}\right.\right.$ $\left.\mathrm{O})\left(\mu_{3}-\mathrm{OH}\right)_{8}\left(\mathrm{H}_{2} \mathrm{O}\right)_{24}\right]^{8+}$, in which the six $\mathrm{Y}^{3+}$ cations are arranged octahedrally around a $\mu_{6}-\mathrm{O}$ atom at the centre of the cationic complex. Each of the eight faces of the $\mathrm{Y}_{6}$ octahedron is capped by an $\mu_{3}-\mathrm{OH}$ group in the form of a distorted cube. In the hexanuclear entity, the $\mathrm{Y}^{3+}$ cations are coordinated by the central $\mu_{6}-\mathrm{O}$ atom, the $\mathrm{O}$ atoms of four $\mu_{3}-\mathrm{OH}$ and of four water molecules. The resulting coordination sphere of the metal ions is a capped square-antiprism. The crystal packing is quite similar to that of the orthorhombic $\left[\operatorname{Ln}_{6}\left(\mu_{6}-\mathrm{O}\right)\left(\mu_{3^{-}}\right.\right.$ $\left.\mathrm{OH})_{8}\left(\mathrm{H}_{2} \mathrm{O}\right)_{24}\right] \mathrm{I}_{8} \cdot 8 \mathrm{H}_{2} \mathrm{O}$ structures with $\mathrm{Ln}=\mathrm{La}-\mathrm{Nd}, \mathrm{Eu}-\mathrm{Tb}$, Dy, except that the title compound exhibits a slight monoclinic distortion. The proximity of the cationic complexes and the lattice water molecules leads to the formation of a three-dimensional hydrogen-bonded network of medium strength.

\section{Chemical context}

Rare-earth-based oxido-hydroxido polynuclear complexes are of interest because of their unique luminescence (Chen et al., 2010; Le Natur et al., 2013; Petit et al., 2009), magnetic properties (Abbas et al., 2010; Xu et al., 2011) or structural characteristics (Zheng, 2001; Andrews et al., 2013). Actually, in this kind of complex, the spatial proximity between metal ions affords cooperative/synergetic effects or energy-transfer mechanisms workable in terms of optical properties. For more than a decade, our group has been involved in the synthesis and the characterization of such rare-earth-based hexanuclear

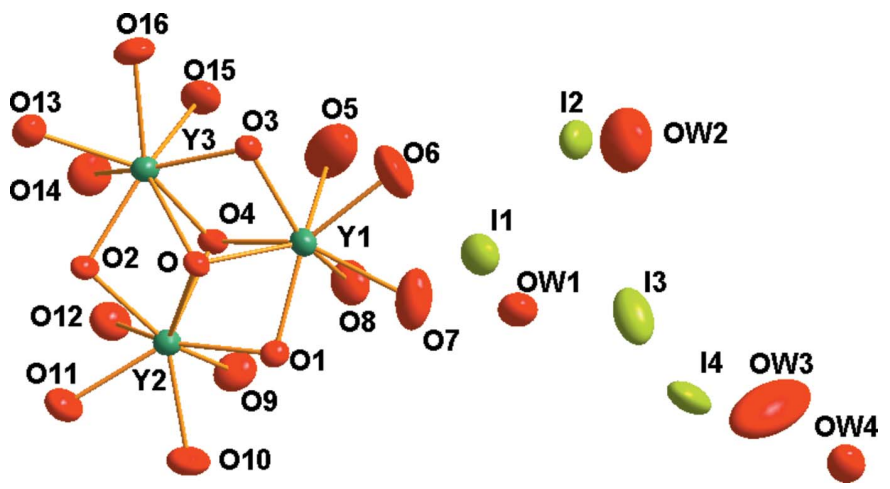

Figure 1

The asymmetric unit of the title complex. Displacement ellipsoids are drawn at the $50 \%$ probability level. 
complexes (Calvez et al., 2010). The hexanuclear complexes crystallize in different structures depending on the counteranion (e.g. nitrate, perchlorate, iodide: Zak et al., 1994; Wang et al., 2000; Mudring et al., 2006), the number of lattice water molecules and/or the radius of the involved lanthanide ion. Since the pioneering work of Zak et al. (1994), we have developed a systematic synthetic procedure for the nitrate counter-anion complex with most of the rare earth elements (Calvez et al., 2008, 2010). In this context, we have undertaken the study of a series of complexes based on the iodide counteranion which have never been obtained with heavier rare earth ions. We report here the synthesis and crystal structure of the yttrium derivative.

\section{Structural commentary}

In contrast to the orthorhombic $\left[\operatorname{Ln}_{6}\left(\mu_{6}-\mathrm{O}\right)\left(\mu_{3}-\mathrm{OH}\right)_{8^{-}}\right.$ $\left.\left(\mathrm{H}_{2} \mathrm{O}\right)_{24}\right] \mathrm{I}_{8} \cdot 8 \mathrm{H}_{2} \mathrm{O}$ structures with $\mathrm{Ln}=\mathrm{La}-\mathrm{Nd}, \mathrm{Eu}-\mathrm{Tb}$, Dy (Mudring \& Babai, 2005; Mudring et al., 2006; Rukk et al., 2009), the crystal structure of the yttrium member of this series has monoclinic symmetry, with the monoclinic angle close to $90^{\circ}$ (Table 2). The asymmetric unit of $\left[\mathrm{Y}_{6}\left(\mu_{6}-\mathrm{O}\right)\left(\mu_{3^{-}}\right.\right.$ $\left.\mathrm{OH})_{8}\left(\mathrm{H}_{2} \mathrm{O}\right)_{24}\right] \mathrm{I}_{8} \cdot 8 \mathrm{H}_{2} \mathrm{O}$ contains half of the formula unit because the complete complex is situated on a centre of inversion. Three independent yttrium cations (Y1, Y2 and $\mathrm{Y} 3)$, four oxygen atoms from $\mu_{3}$-hydroxyl groups $(\mathrm{O} 1, \mathrm{O} 2, \mathrm{O} 3$, $\mathrm{O} 4)$, twelve oxygen atoms of terminal aqua ligands coordinating to each yttrium cation (Y1: O5, O6, O7, O8; Y2: O9, O10, O11, O12; Y3: O13, O14, O15 O16), one $\mu_{6}$-bridging $\mathrm{O}$ atom (O) lying on an inversion centre, four iodide anions (I1, I2, I3, I4) and four oxygen atoms of lattice water molecules ( $\mathrm{OW} 1$, $\mathrm{OW}$, OW3, OW4) are present in the crystal structure (Fig. 1). Calculations with the SHAPE software suite (Alvarez et al., 2005) indicate that each of the coordination polyhedra surrounding the $\mathrm{Y}^{3+}$ ions is best described as a spherical capped square-antiprism (Ruiz-Martínez et al., 2010) with idealized $C_{4 v}$ symmetry. However, the true symmetry of this structural fragment in the title structure is $C_{1}$.

Since the $\mu_{6}-\mathrm{O}$ atom is located on an inversion centre and binds to six $\mathrm{Y}^{3+}$ cations, a slightly distorted anion-centred $\left[\mathrm{OY}_{6}\right]$ octahedron results (Fig. 2). The average of the Y $\cdots \mathrm{Y}$

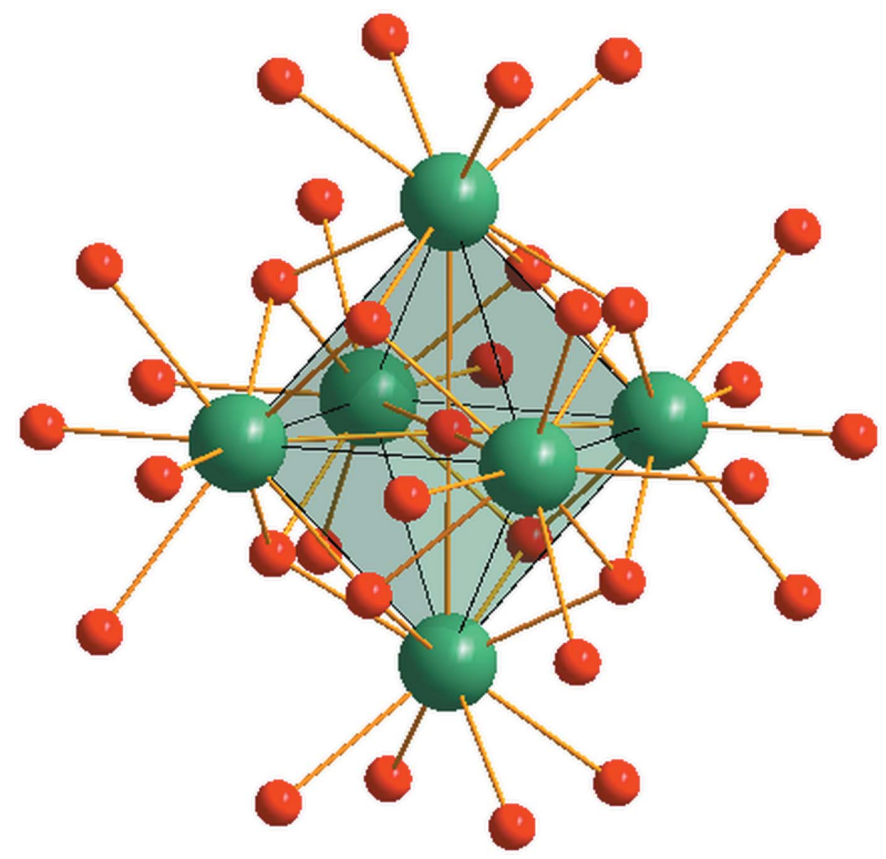

Figure 2

The $\mathrm{OY}_{6}$ octahedron in the complex $\left[\mathrm{Y}_{6}\left(\mu_{6}-\mathrm{O}\right)\left(\mu_{3}-\mathrm{OH}\right)_{8}\left(\mathrm{H}_{2} \mathrm{O}\right)_{24}\right]^{8+}$ cation. $\mathrm{Y}$ atoms are green and $\mathrm{O}$ atoms are red.

distances between adjacent cations in the octahedron is found to be $3.536 \AA$. The mean $\mathrm{Y}-\left(\mu_{6}-\mathrm{O}\right)$ distance is $2.537 \AA$, while the averaged $\mathrm{Y}-\left(\mu_{3}-\mathrm{OH}\right)$ is $2.34 \AA$. The hydroxide ions are situated above the eight faces of the $\mathrm{OY}_{6}$ octahedron and form a distorted cube around the octahedron (Fig. 2).

\section{Supramolecular features}

The hexanuclear $\left[\mathrm{Y}_{6}\left(\mu_{6}-\mathrm{O}\right)\left(\mu_{3}-\mathrm{OH}\right)_{8}\left(\mathrm{H}_{2} \mathrm{O}\right)_{24}\right]^{8+}$ units are arranged in a body-centred fashion in the crystal structure. Each of these units is surrounded by twelve iodide anions, connecting the units to each other through Coulombic interactions. Although the hydrogen atoms of the water molecules and hydroxide groups could not be located, the range of $\mathrm{O} \cdots \mathrm{O}$ distances between the cationic complex and the lattice water molecules suggest the formation of medium-strength
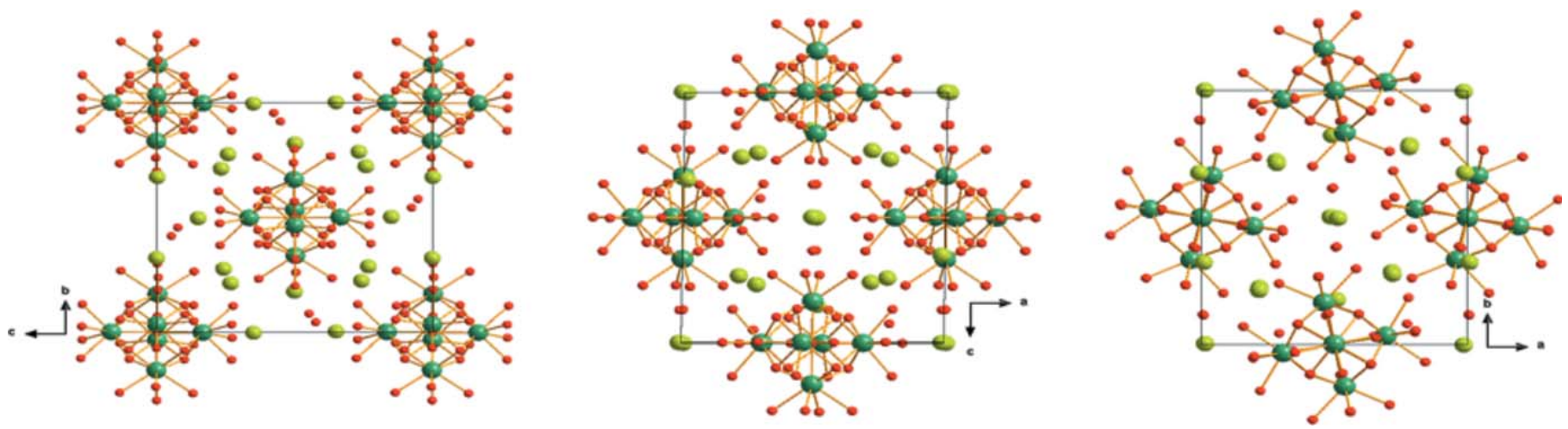

Figure 3

The crystal structure of $\left[\mathrm{Y}_{6}\left(\mu_{6}-\mathrm{O}\right)\left(\mu_{3}-\mathrm{OH}\right)_{8}\left(\mathrm{H}_{2} \mathrm{O}\right)_{24}\right] \mathrm{I}_{8} \cdot 8 \mathrm{H}_{2} \mathrm{O}$ in projections along [100], [010] and [001], respectively, from left to right. $\mathrm{Y}$ atoms are green, $\mathrm{O}$ atoms are red and $\mathrm{I}$ atoms are yellow. 
Table 1

Hydrogen-bond geometry $(\AA)$.

\begin{tabular}{|c|c|}
\hline$D \cdots A$ & $D \cdots A$ \\
\hline $\mathrm{O} 7 \ldots \mathrm{OW} 2$ & $2.646(4)$ \\
\hline $\mathrm{O} 10 \cdots \mathrm{OW} 3$ & $2.764(1)$ \\
\hline $\mathrm{O} 13 \cdots \mathrm{OW} 4$ & $2.803(8)$ \\
\hline $\mathrm{O} 15 \cdots \mathrm{OW} 1$ & $2.767(2)$ \\
\hline O16 .. OW4 & $2.836(2)$ \\
\hline $\mathrm{O} 16 \cdots \mathrm{O} W 1$ & $2.851(6)$ \\
\hline
\end{tabular}

hydrogen bonds (Table 1). These interactions lead to the formation of a three-dimensional network in the structure (Fig. 3).

\section{Synthesis and crystallization}

Yttrium oxide $\mathrm{Y}_{2} \mathrm{O}_{3}(2 \mathrm{~g}$, Strem Chemicals $4 \mathrm{M})$ was dissolved in fresh hydroiodic acid $(9 \mathrm{ml}, 57 \mathrm{wt} \%$, unstabilized from Acros Organics) under gentle heating (323 K). If the acid used is not fresh, it should be distilled twice. The clear solution was exposed to air under isothermal conditions (6 weeks). At this stage, the $\mathrm{pH}$ of the solution remains acidic. Large pale-yellow polyhedral crystals were separated manually from the solution and were mounted into a glass capillary.

\section{Refinement}

Crystal data, data collection and structure refinement details are summarized in Table 2. The hydrogen atoms from the water molecules or hydroxide could not be assigned reliably and thus were not included in the refinement. However, they were taken into account for the chemical formula sum, moiety, weight, as well as for the absorption coefficient and the number of electrons in the unit cell.

\section{Acknowledgements}

The Centre de DIFractométrie X of the University of Rennes 1 is acknowledged for the data collection. FLeN thanks Région Bretagne for funding (ARED Ln6 Program).

\section{References}

Abbas, G., Lan, Y., Kostakis, G. E., Wernsdorfer, W., Anson, C. E. \& Powell, A. K. (2010). Inorg. Chem. 49, 8067-8072.

Alvarez, S., Alemany, P., Casanova, D., Cirera, J., Llunell, M. \& Avnir, D. (2005). Coord. Chem. Rev. 249, 1693-1708.

Andrews, P. C., Gee, W. J., Junk, P. C. \& Massi, M. (2013). New J. Chem. 37, 35-48.

Brandenburg, K. (2006). DIAMOND. Crystal Impact GbR, Bonn, Germany.

Calvez, G., Daiguebonne, C., Guillou, O., Pott, T., Méléard, P. \& Le Dret, F. (2010). C. R. Chim. 13, 715-730.

Calvez, G., Guillou, O., Daiguebonne, C., Car, P. E., Guillerm, V., Gérault, Y., Le Dret, F. \& Mahé, N. (2008). Inorg. Chim. Acta, 361, 2349-2356.
Table 2

Experimental details.

\begin{tabular}{|c|c|}
\hline Crystal data & \\
\hline Chemical formula & {$\left[\mathrm{Y}_{6} \mathrm{O}(\mathrm{OH})_{8}\left(\mathrm{H}_{2} \mathrm{O}\right)_{24}\right] \mathrm{I}_{8} \cdot 8 \mathrm{H}_{2} \mathrm{O}$} \\
\hline$M_{\mathrm{r}}$ & 2277.24 \\
\hline Crystal system, space group & Monoclinic, $P 2_{1} / n$ \\
\hline Temperature $(\mathrm{K})$ & 293 \\
\hline$a, b, c(\AA)$ & $\begin{array}{l}12.9099(2), 14.8050(2), \\
14.7933(3)\end{array}$ \\
\hline$\beta\left(^{\circ}\right)$ & $90.821(1)$ \\
\hline$V\left(\AA^{3}\right)$ & $2827.17(8)$ \\
\hline$Z$ & 2 \\
\hline Radiation type & Mo $K \alpha$ \\
\hline$\mu\left(\mathrm{mm}^{-1}\right)$ & 10.54 \\
\hline Crystal size $(\mathrm{mm})$ & $0.18 \times 0.14 \times 0.1$ \\
\hline Data collection & \\
\hline Diffractometer & Nonius KappaCCD \\
\hline Absorption correction & Gaussian (Coppens et al., 1965) \\
\hline$T_{\min }, T_{\max }$ & $0.018,0.091$ \\
\hline $\begin{array}{l}\text { No. of measured, independent and } \\
\text { observed }[I>2 \sigma(I)] \text { reflections }\end{array}$ & $35352,6374,5449$ \\
\hline$R_{\text {int }}$ & 0.124 \\
\hline$(\sin \theta / \lambda)_{\max }\left(\AA^{-1}\right)$ & 0.647 \\
\hline Refinement & \\
\hline$R\left[F^{2}>2 \sigma\left(F^{2}\right)\right], w R\left(F^{2}\right), S$ & $0.067,0.178,1.11$ \\
\hline No. of reflections & 6374 \\
\hline No. of parameters & 251 \\
\hline & $\begin{array}{l}w=1 /\left[\sigma^{2}\left(F_{\mathrm{o}}^{2}\right)+(0.0487 P)^{2}+\right. \\
\quad 43.8859 P] \text { where } P=\left(F_{\mathrm{o}}^{2}+\right. \\
\left.\quad 2 F_{\mathrm{c}}^{2}\right) / 3\end{array}$ \\
\hline$\Delta \rho_{\max }, \Delta \rho_{\min }\left(\mathrm{e} \AA^{-3}\right)$ & $2.62,-1.83$ \\
\hline
\end{tabular}

Computer programs: COLLECT (Nonius, 1998), EVALCCD (Duisenberg et al., 2003), SHELXS97 and SHELXL97 (Sheldrick, 2008), DIAMOND (Brandenburg, 2006) and publCIF (Westrip, 2010).

Chen, X.-Y., Yang, X. \& Holliday, B. J. (2010). Inorg. Chem. 49, $2583-$ 2585.

Coppens, P., Leiserowitz, L. \& Rabinovich, D. (1965). Acta Cryst. 18, $1035-1038$

Duisenberg, A. J. M., Kroon-Batenburg, L. M. J. \& Schreurs, A. M. M. (2003). J. Appl. Cryst. 36, 220-229.

Le Natur, F., Calvez, G., Daiguebonne, C., Guillou, G., Bernot, K., Ledoux, J., Le Pollès, L. \& Roiland, C. (2013). Inorg. Chem. 52, 6720-6730.

Mudring, A.-V. \& Babai, A. (2005). Z. Anorg. Allg. Chem. 631, 261263.

Mudring, A.-V., Timofte, T. \& Babai, A. (2006). Inorg. Chem. 45, $5162-5166$.

Nonius (1998). COLLECT. Nonius BV, Delft, The Netherlands.

Petit, S., Baril-Robert, F., Pilet, G., Reber, C. \& Luneau, D. (2009). Dalton Trans. pp. 6809-6815.

Ruiz-Martínez, A., Casanova, D. \& Alvarez, S. (2010). Chem. Eur. J. 16, 6567-6581.

Rukk, N. S., Al'bov, D. V., Skryabina, A. Y., Osipov, R. A. \& Alikberova, L. Y. (2009). Russ. J. Coord. Chem. 35, 12-14.

Sheldrick, G. M. (2008). Acta Cryst. A64, 112-122.

Wang, R., Carducci, M. D. \& Zheng, Z. (2000). Inorg. Chem. 39, 18361837.

Westrip, S. P. (2010). J. Appl. Cryst. 43, 920-925.

Xu, X., Zhao, L., Xu, G.-F., Guo, Y.-N., Tang, J. \& Liu, Z. (2011). Dalton Trans. 40, 6440-6444.

Zak, Z., Unfried, P. \& Giester, G. (1994). J. Alloys Compd, 205, 235242.

Zheng, Z. (2001). Chem. Commun. pp. 2521-2529. 


\section{supporting information}

Acta Cryst. (2014). E70, 577-579 [doi:10.1107/S1600536814025434]

\section{Crystal structure of $\left.\left[\mathrm{Y}_{6}\left(\mu_{6}-\mathrm{O}\right)\left(\mu_{3}-\mathrm{OH}\right)_{8}\left(\mathrm{H}_{2} \mathrm{O}\right)_{24}\right]\right]_{8} \cdot 8 \mathrm{H}_{2} \mathrm{O}$}

\section{François Le Natur, Guillaume Calvez, Olivier Guillou, Carole Daiguebonne and Kevin Bernot}

\section{Computing details}

Data collection: COLLECT (Nonius, 1998); cell refinement: COLLECT (Nonius, 1998); data reduction: EVALCCD (Duisenberg et al., 2003); program(s) used to solve structure: SHELXS97 (Sheldrick, 2008); program(s) used to refine structure: SHELXL97 (Sheldrick, 2008); molecular graphics: DIAMOND (Brandenburg, 2006); software used to prepare material for publication: publCIF (Westrip, 2010).

Octa- $\mu_{3}$-hydroxido- $\mu_{6}$-oxido-hexakis[tetraaquayttrium(III)] octaiodide octahydrate

Crystal data

$\left[\mathrm{Y}_{6} \mathrm{O}(\mathrm{OH})_{8}\left(\mathrm{H}_{2} \mathrm{O}\right)_{24}\right] \mathrm{I}_{8} \cdot 8 \mathrm{H}_{2} \mathrm{O}$

$F(000)=2116$

$M_{r}=2277.24$

Monoclinic, $P 2_{1} / n$

Hall symbol: -P 2yn

$a=12.9099$ (2) $\AA$

$b=14.8050(2) \AA$

$c=14.7933(3) \AA$

$\beta=90.821(1)^{\circ}$

$V=2827.17(8) \AA^{3}$

$Z=2$

$D_{\mathrm{x}}=2.675 \mathrm{Mg} \mathrm{m}^{-3}$

Mo $K \alpha$ radiation, $\lambda=0.71073 \AA$

Cell parameters from 62388 reflections

$\theta=2.9-27.5^{\circ}$

$\mu=10.54 \mathrm{~mm}^{-1}$

$T=293 \mathrm{~K}$

Block, colorless

$0.18 \times 0.14 \times 0.1 \mathrm{~mm}$

\section{Data collection}

Nonius KappaCCD diffractometer

Radiation source: fine-focus sealed tube Graphite monochromator

CCD rotation images, thick slices scans

Absorption correction: gaussian

(Coppens et al., 1965)

$T_{\min }=0.018, T_{\max }=0.091$

35352 measured reflections

6374 independent reflections

5449 reflections with $I>2 \sigma(I)$

$R_{\text {int }}=0.124$

$\theta_{\max }=27.4^{\circ}, \theta_{\min }=3.1^{\circ}$

$h=-16 \rightarrow 16$

$k=-19 \rightarrow 18$

$l=-19 \rightarrow 19$

\section{Refinement}

Refinement on $F^{2}$

Least-squares matrix: full

$R\left[F^{2}>2 \sigma\left(F^{2}\right)\right]=0.067$

$w R\left(F^{2}\right)=0.178$

$S=1.11$

6374 reflections

251 parameters

0 restraints

Primary atom site location: structure-invariant direct methods

Secondary atom site location: difference Fourier

$$
\text { map }
$$

$w=1 /\left[\sigma^{2}\left(F_{\mathrm{o}}{ }^{2}\right)+(0.0487 P)^{2}+43.8859 P\right]$

where $P=\left(F_{\mathrm{o}}^{2}+2 F_{\mathrm{c}}^{2}\right) / 3$

$(\Delta / \sigma)_{\max }=0.001$

$\Delta \rho_{\max }=2.62 \mathrm{e} \AA^{-3}$

$\Delta \rho_{\min }=-1.83$ e $\AA^{-3}$

Extinction correction: SHELXL97 (Sheldrick, 2008), $\mathrm{Fc}^{*}=\mathrm{kFc}\left[1+0.001 \mathrm{xFc}^{2} \lambda^{3} / \sin (2 \theta)\right]^{-1 / 4}$

Extinction coefficient: 0.00258 (19) 


\section{Special details}

Experimental. 6336 sampling points

Geometry. All s.u.'s (except the s.u. in the dihedral angle between two 1.s. planes) are estimated using the full covariance matrix. The cell s.u.'s are taken into account individually in the estimation of s.u.'s in distances, angles and torsion angles; correlations between s.u.'s in cell parameters are only used when they are defined by crystal symmetry. An approximate (isotropic) treatment of cell s.u.'s is used for estimating s.u.'s involving l.s. planes.

Refinement. Refinement of $F^{2}$ against ALL reflections. The weighted $R$-factor $w R$ and goodness of fit $S$ are based on $F^{2}$, conventional $R$-factors $R$ are based on $F$, with $F$ set to zero for negative $F^{2}$. The threshold expression of $F^{2}>2 \sigma\left(F^{2}\right)$ is used only for calculating $R$-factors(gt) etc. and is not relevant to the choice of reflections for refinement. $R$-factors based on $F^{2}$ are statistically about twice as large as those based on $F$, and $R$ - factors based on ALL data will be even larger.

Fractional atomic coordinates and isotropic or equivalent isotropic displacement parameters $\left(\AA^{2}\right)$

\begin{tabular}{|c|c|c|c|c|}
\hline & $x$ & $y$ & $z$ & $U_{\text {iso }} * / U_{\text {eq }}$ \\
\hline Y1 & $0.50083(7)$ & $1.00150(6)$ & $1.16560(6)$ & $0.0335(2)$ \\
\hline Y2 & $0.46090(7)$ & $1.16586(6)$ & $0.99794(6)$ & $0.0317(2)$ \\
\hline Y3 & $0.30677(7)$ & $0.96613(6)$ & $0.99944(6)$ & $0.0323(2)$ \\
\hline I1 & $0.49148(9)$ & $0.82346(8)$ & $0.49868(6)$ & $0.0707(3)$ \\
\hline $\mathrm{I} 2$ & $0.28137(7)$ & $0.72379(7)$ & $0.24171(7)$ & $0.0650(3)$ \\
\hline $\mathrm{I} 3$ & $0.48625(14)$ & $0.50289(7)$ & $0.14980(8)$ & $0.0932(4)$ \\
\hline I4 & $0.78359(8)$ & $0.78678(8)$ & $0.26729(8)$ & $0.0792(4)$ \\
\hline $\mathrm{O}$ & 0.5000 & 1.0000 & 1.0000 & $0.0293(17)$ \\
\hline $\mathrm{O} 1$ & $0.3633(5)$ & $1.0806(4)$ & 1.0999 (4) & $0.0328(13)$ \\
\hline $\mathrm{O} 2$ & $0.4097(5)$ & $0.8826(4)$ & $1.1002(4)$ & $0.0313(13)$ \\
\hline $\mathrm{O} 3$ & $0.3622(5)$ & $1.0773(4)$ & $0.8978(4)$ & $0.0321(13)$ \\
\hline $\mathrm{O} 4$ & $0.4081(5)$ & $0.8803(4)$ & $0.9008(4)$ & $0.0335(13)$ \\
\hline O5 & $0.6563(13)$ & $1.0251(17)$ & $1.2721(11)$ & $0.150(8)$ \\
\hline O6 & $0.5306(14)$ & $0.8859(13)$ & $1.2758(10)$ & $0.136(7)$ \\
\hline $\mathrm{O} 7$ & $0.3511(12)$ & $0.9672(11)$ & $1.2711(9)$ & $0.106(5)$ \\
\hline O8 & $0.4775(9)$ & $1.1210(7)$ & $1.2708(6)$ & 0.069 (3) \\
\hline O9 & $0.4402(8)$ & $1.2676(6)$ & $1.1305(7)$ & $0.064(2)$ \\
\hline O10 & $0.2918(7)$ & $1.2392(6)$ & $0.9958(7)$ & $0.065(2)$ \\
\hline $\mathrm{O} 11$ & $0.4402(7)$ & $1.2644(6)$ & $0.8649(7)$ & $0.060(2)$ \\
\hline $\mathrm{O} 12$ & $0.5819(8)$ & $1.2981(6)$ & $0.9988(7)$ & $0.066(2)$ \\
\hline $\mathrm{O} 13$ & $0.1968(6)$ & $0.9524(7)$ & $1.1339(6)$ & $0.058(2)$ \\
\hline $\mathrm{O} 14$ & $0.2216(8)$ & $0.8163(7)$ & $1.0027(8)$ & 0.075 \\
\hline $\mathrm{O} 15$ & $0.1950(6)$ & $0.9452(7)$ & $0.8664(6)$ & $0.059(2)$ \\
\hline O16 & $0.1578(7)$ & $1.0700(7)$ & $0.9987(7)$ & $0.063(2)$ \\
\hline OW1 & $-0.0079(7)$ & $0.8851(8)$ & $0.8689(7)$ & 0.070 \\
\hline OW2 & $0.2083(19)$ & $0.5431(17)$ & $0.0762(13)$ & $0.164(8)$ \\
\hline OW3 & $0.7261(18)$ & $0.5806(14)$ & $0.051(2)$ & $0.216(13)$ \\
\hline OW4 & $0.9948(7)$ & $0.8828(8)$ & $0.1328(7)$ & 0.069 \\
\hline
\end{tabular}

Atomic displacement parameters $\left(\AA^{2}\right)$

\begin{tabular}{lllllll}
\hline & $U^{11}$ & $U^{22}$ & $U^{33}$ & $U^{12}$ & $U^{13}$ & $U^{23}$ \\
\hline Y1 & $0.0380(5)$ & $0.0307(5)$ & $0.0318(4)$ & $0.0014(3)$ & $-0.0006(3)$ & $0.0000(3)$ \\
Y2 & $0.0313(4)$ & $0.0271(4)$ & $0.0367(5)$ & $-0.0001(3)$ & $-0.0010(3)$ & $0.0003(3)$ \\
Y3 & $0.0297(4)$ & $0.0292(4)$ & $0.0381(5)$ & $-0.0001(3)$ & $-0.0011(3)$ & $0.0003(3)$
\end{tabular}




\begin{tabular}{lllllll} 
I1 & $0.0799(7)$ & $0.0740(7)$ & $0.0580(5)$ & $0.0010(5)$ & $-0.0028(4)$ & $-0.0014(4)$ \\
I2 & $0.0624(5)$ & $0.0599(5)$ & $0.0731(6)$ & $-0.0069(4)$ & $0.0146(4)$ & $0.0202(4)$ \\
I3 & $0.1624(13)$ & $0.0473(5)$ & $0.0701(7)$ & $-0.0080(6)$ & $0.0074(7)$ & $0.0003(4)$ \\
I4 & $0.0647(6)$ & $0.0771(7)$ & $0.0950(8)$ & $0.0062(5)$ & $-0.0237(5)$ & $0.0377(6)$ \\
O & $0.035(4)$ & $0.021(4)$ & $0.032(4)$ & $0.001(3)$ & $-0.002(3)$ & $0.002(3)$ \\
O1 & $0.032(3)$ & $0.028(3)$ & $0.039(3)$ & $-0.001(2)$ & $0.001(3)$ & $-0.003(3)$ \\
O2 & $0.029(3)$ & $0.023(3)$ & $0.042(3)$ & $0.001(2)$ & $0.000(3)$ & $0.005(3)$ \\
O3 & $0.029(3)$ & $0.033(3)$ & $0.034(3)$ & $-0.002(2)$ & $-0.003(2)$ & $0.003(3)$ \\
O4 & $0.029(3)$ & $0.033(3)$ & $0.038(3)$ & $0.000(2)$ & $-0.006(3)$ & $0.000(3)$ \\
O5 & $0.099(11)$ & $0.26(3)$ & $0.090(11)$ & $0.009(13)$ & $-0.001(9)$ & $-0.023(13)$ \\
O6 & $0.157(15)$ & $0.171(17)$ & $0.080(9)$ & $0.027(12)$ & $0.002(9)$ & $0.065(10)$ \\
O7 & $0.127(11)$ & $0.120(11)$ & $0.070(7)$ & $-0.043(9)$ & $0.026(7)$ & $-0.005(7)$ \\
O8 & $0.101(8)$ & $0.057(6)$ & $0.050(5)$ & $0.002(5)$ & $0.000(5)$ & $-0.018(4)$ \\
O9 & $0.064(6)$ & $0.051(5)$ & $0.078(6)$ & $-0.003(4)$ & $0.004(5)$ & $-0.020(5)$ \\
O10 & $0.054(5)$ & $0.052(5)$ & $0.089(7)$ & $0.018(4)$ & $-0.004(5)$ & $0.006(5)$ \\
O11 & $0.058(5)$ & $0.047(5)$ & $0.075(6)$ & $0.001(4)$ & $-0.006(4)$ & $0.020(4)$ \\
O12 & $0.067(6)$ & $0.048(5)$ & $0.082(7)$ & $-0.007(4)$ & $-0.004(5)$ & $0.007(5)$ \\
O13 & $0.041(4)$ & $0.073(6)$ & $0.061(5)$ & $0.000(4)$ & $0.005(4)$ & $0.010(4)$ \\
O14 & $0.062(6)$ & $0.065(6)$ & $0.098(8)$ & $-0.027(5)$ & $-0.003(5)$ & $0.002(6)$ \\
O15 & $0.045(4)$ & $0.075(6)$ & $0.058(5)$ & $-0.004(4)$ & $-0.018(4)$ & $-0.005(4)$ \\
O16 & $0.041(4)$ & $0.079(6)$ & $0.071(6)$ & $0.023(4)$ & $0.002(4)$ & $-0.006(5)$ \\
OW1 & $0.051(5)$ & $0.086(7)$ & $0.072(6)$ & $-0.006(5)$ & $-0.004(4)$ & $-0.009(5)$ \\
OW2 & $0.19(2)$ & $0.19(2)$ & $0.114(14)$ & $0.012(17)$ & $0.028(13)$ & $-0.005(14)$ \\
OW3 & $0.158(19)$ & $0.091(13)$ & $0.40(4)$ & $0.027(13)$ & $0.09(2)$ & $0.004(19)$ \\
OW4 & $0.052(5)$ & $0.086(7)$ & $0.068(6)$ & $-0.010(5)$ & $-0.006(4)$ & $0.010(5)$ \\
& & & & & & \\
\hline
\end{tabular}

Geometric parameters $\left(\AA,{ }^{\circ}\right)$

\begin{tabular}{llll}
\hline $\mathrm{Y} 1-\mathrm{O} 2$ & $2.321(6)$ & $\mathrm{Y} 2-\mathrm{O} 11$ & $2.462(9)$ \\
$\mathrm{Y} 1-\mathrm{O} 3^{\mathrm{i}}$ & $2.326(6)$ & $\mathrm{Y} 2-\mathrm{O} 9$ & $2.490(9)$ \\
$\mathrm{Y} 1-\mathrm{O} 1$ & $2.328(7)$ & $\mathrm{Y} 2-\mathrm{O} 12$ & $2.506(9)$ \\
$\mathrm{Y} 1-\mathrm{O} 4^{\mathrm{i}}$ & $2.332(7)$ & $\mathrm{Y} 2-\mathrm{O}$ & $2.5070(9)$ \\
$\mathrm{Y} 1-\mathrm{O} 8$ & $2.378(9)$ & $\mathrm{Y} 3-\mathrm{O} 2$ & $2.336(6)$ \\
$\mathrm{Y} 1-\mathrm{O} 6$ & $2.390(14)$ & $\mathrm{Y} 3-\mathrm{O} 3$ & $2.347(6)$ \\
$\mathrm{Y} 1-\mathrm{O}$ & $2.4497(9)$ & $\mathrm{Y} 3-\mathrm{O} 4$ & $2.348(7)$ \\
$\mathrm{Y} 1-\mathrm{O} 7$ & $2.553(13)$ & $\mathrm{Y} 3-\mathrm{O} 1$ & $2.362(6)$ \\
$\mathrm{Y} 1-\mathrm{O} 5$ & $2.557(18)$ & $\mathrm{Y} 3-\mathrm{O} 15$ & $2.443(8)$ \\
$\mathrm{Y} 2-\mathrm{O} 2^{\mathrm{i}}$ & $2.341(6)$ & $\mathrm{Y} 3-\mathrm{O} 16$ & $2.462(8)$ \\
$\mathrm{Y} 2-\mathrm{O} 3$ & $\mathrm{Y} 3-\mathrm{O} 13$ & $2.469(8)$ \\
$\mathrm{Y} 2-\mathrm{O} 4^{\mathrm{i}}$ & $2.341(6)$ & $\mathrm{Y} 3-\mathrm{O} 14$ & $2.477(10)$ \\
$\mathrm{Y} 2-\mathrm{O} 1$ & $2.344(6)$ & $\mathrm{Y} 3-\mathrm{O}$ & $2.5444(9)$ \\
$\mathrm{Y} 2-\mathrm{O} 10$ & $2.347(6)$ & & \\
& $2.438(9)$ & $\mathrm{O} 10-\mathrm{Y} 2-\mathrm{O}$ & $128.0(2)$ \\
$\mathrm{O} 2-\mathrm{Y} 1-\mathrm{O} 3^{\mathrm{i}}$ & & $\mathrm{O} 11-\mathrm{Y} 2-\mathrm{O}$ & $127.6(2)$ \\
$\mathrm{O} 2-\mathrm{Y} 1-\mathrm{O} 1$ & $80.5(2)$ & $\mathrm{O} 9-\mathrm{Y} 2-\mathrm{O}$ & $127.3(3)$ \\
$\mathrm{O} 3-\mathrm{Y} 1-\mathrm{O} 1$ & $131.5(2)$ & $\mathrm{O} 12-\mathrm{Y} 2-\mathrm{O}$ & $127.1(2)$ \\
$\mathrm{O} 2-\mathrm{Y} 1-\mathrm{O} 4^{\mathrm{i}}$ & $130.4(2)$ & $\mathrm{O} 2-\mathrm{Y} 3-\mathrm{O} 3$ & \\
$\mathrm{O} 3-\mathrm{Y} 1-\mathrm{O} 4^{\mathrm{i}}$ & $79.4(2)$ &
\end{tabular}




\begin{tabular}{|c|c|c|c|}
\hline $\mathrm{O} 1-\mathrm{Y} 1-\mathrm{O} 4^{\mathrm{i}}$ & $80.4(2)$ & $\mathrm{O} 2-\mathrm{Y} 3-\mathrm{O} 4$ & $78.1(2)$ \\
\hline $\mathrm{O} 2-\mathrm{Y} 1-\mathrm{O} 8$ & $140.2(3)$ & $\mathrm{O} 3-\mathrm{Y} 3-\mathrm{O} 4$ & $78.7(2)$ \\
\hline $\mathrm{O} 3{ }^{\mathrm{i}}-\mathrm{Y} 1-\mathrm{O} 8$ & $137.8(3)$ & $\mathrm{O} 2-\mathrm{Y} 3-\mathrm{O} 1$ & $79.1(2)$ \\
\hline $\mathrm{O} 1-\mathrm{Y} 1-\mathrm{O} 8$ & $78.2(3)$ & $\mathrm{O} 3-\mathrm{Y} 3-\mathrm{O} 1$ & $78.8(2)$ \\
\hline $\mathrm{O} 4 \mathrm{i}-\mathrm{Y} 1-\mathrm{O} 8$ & $77.7(3)$ & $\mathrm{O} 4-\mathrm{Y} 3-\mathrm{O} 1$ & $127.5(2)$ \\
\hline $\mathrm{O} 2-\mathrm{Y} 1-\mathrm{O} 6$ & $79.4(5)$ & $\mathrm{O} 2-\mathrm{Y} 3-\mathrm{O} 15$ & $140.4(3)$ \\
\hline $\mathrm{O} 3-\mathrm{i}-\mathrm{Y} 1-\mathrm{O} 6$ & $78.5(5)$ & $\mathrm{O} 3-\mathrm{Y} 3-\mathrm{O} 15$ & $75.8(3)$ \\
\hline $\mathrm{O} 1-\mathrm{Y} 1-\mathrm{O} 6$ & $139.4(5)$ & $\mathrm{O} 4-\mathrm{Y} 3-\mathrm{O} 15$ & $76.0(3)$ \\
\hline $\mathrm{O} 4^{\mathrm{i}}-\mathrm{Y} 1-\mathrm{O} 6$ & $138.4(5)$ & $\mathrm{O} 1-\mathrm{Y} 3-\mathrm{O} 15$ & $140.5(3)$ \\
\hline $\mathrm{O} 8-\mathrm{Y} 1-\mathrm{O} 6$ & $96.2(6)$ & $\mathrm{O} 2-\mathrm{Y} 3-\mathrm{O} 16$ & $140.4(3)$ \\
\hline $\mathrm{O} 2-\mathrm{Y} 1-\mathrm{O}$ & $65.23(16)$ & $\mathrm{O} 3-\mathrm{Y} 3-\mathrm{O} 16$ & $78.7(3)$ \\
\hline $\mathrm{O}^{\mathrm{i}}-\mathrm{Y} 1-\mathrm{O}$ & $65.46(16)$ & $\mathrm{O} 4-\mathrm{Y} 3-\mathrm{O} 16$ & $141.0(3)$ \\
\hline $\mathrm{O} 1-\mathrm{Y} 1-\mathrm{O}$ & $66.07(16)$ & $\mathrm{O} 1-\mathrm{Y} 3-\mathrm{O} 16$ & $77.8(3)$ \\
\hline $\mathrm{O} 4-\mathrm{Y} 1-\mathrm{O}$ & $65.21(16)$ & $\mathrm{O} 15-\mathrm{Y} 3-\mathrm{O} 16$ & $67.9(3)$ \\
\hline $\mathrm{O} 8-\mathrm{Y} 1-\mathrm{O}$ & $131.5(3)$ & $\mathrm{O} 2-\mathrm{Y} 3-\mathrm{O} 13$ & $76.8(3)$ \\
\hline $\mathrm{O} 6-\mathrm{Y} 1-\mathrm{O}$ & $132.4(5)$ & $\mathrm{O} 3-\mathrm{Y} 3-\mathrm{O} 13$ & $139.3(3)$ \\
\hline $\mathrm{O} 2-\mathrm{Y} 1-\mathrm{O} 7$ & $73.7(4)$ & $\mathrm{O} 4-\mathrm{Y} 3-\mathrm{O} 13$ & $142.0(3)$ \\
\hline $\mathrm{O} 3{ }^{\mathrm{i}}-\mathrm{Y} 1-\mathrm{O} 7$ & $137.2(4)$ & $\mathrm{O} 1-\mathrm{Y} 3-\mathrm{O} 13$ & $74.2(3)$ \\
\hline $\mathrm{O} 1-\mathrm{Y} 1-\mathrm{O} 7$ & $77.0(4)$ & $\mathrm{O} 15-\mathrm{Y} 3-\mathrm{O} 13$ & $107.4(3)$ \\
\hline $\mathrm{O} 4-\mathrm{i} 1-\mathrm{O} 7$ & $142.9(4)$ & $\mathrm{O} 16-\mathrm{Y} 3-\mathrm{O} 13$ & $66.2(3)$ \\
\hline $\mathrm{O} 8-\mathrm{Y} 1-\mathrm{O} 7$ & $69.2(4)$ & $\mathrm{O} 2-\mathrm{Y} 3-\mathrm{O} 14$ & $76.3(3)$ \\
\hline $\mathrm{O} 6-\mathrm{Y} 1-\mathrm{O} 7$ & $63.7(6)$ & $\mathrm{O} 3-\mathrm{Y} 3-\mathrm{O} 14$ & $141.2(3)$ \\
\hline $\mathrm{O}-\mathrm{Y} 1-\mathrm{O} 7$ & $128.1(3)$ & $\mathrm{O} 4-\mathrm{Y} 3-\mathrm{O} 14$ & $77.2(3)$ \\
\hline $\mathrm{O} 2-\mathrm{Y} 1-\mathrm{O} 5$ & $138.5(6)$ & $\mathrm{O} 1-\mathrm{Y} 3-\mathrm{O} 14$ & $139.8(3)$ \\
\hline $\mathrm{O} 3^{\mathrm{i}}-\mathrm{Y} 1-\mathrm{O} 5$ & $73.9(4)$ & $\mathrm{O} 15-\mathrm{Y} 3-\mathrm{O} 14$ & $69.2(4)$ \\
\hline $\mathrm{O} 1-\mathrm{Y} 1-\mathrm{O} 5$ & $140.9(6)$ & $\mathrm{O} 16-\mathrm{Y} 3-\mathrm{O} 14$ & $102.3(4)$ \\
\hline $\mathrm{O} 44^{\mathrm{i}-\mathrm{Y} 1-\mathrm{O} 5}$ & $76.2(5)$ & $\mathrm{O} 13-\mathrm{Y} 3-\mathrm{O} 14$ & $69.5(4)$ \\
\hline $\mathrm{O} 8-\mathrm{Y} 1-\mathrm{O} 5$ & $66.5(5)$ & $\mathrm{O} 2-\mathrm{Y} 3-\mathrm{O}$ & $63.48(15)$ \\
\hline $\mathrm{O} 6-\mathrm{Y} 1-\mathrm{O} 5$ & $64.0(7)$ & $\mathrm{O} 3-\mathrm{Y} 3-\mathrm{O}$ & $63.63(15)$ \\
\hline $\mathrm{O}-\mathrm{Y} 1-\mathrm{O} 5$ & $127.5(4)$ & $\mathrm{O} 4-\mathrm{Y} 3-\mathrm{O}$ & $63.47(15)$ \\
\hline $\mathrm{O} 7-\mathrm{Y} 1-\mathrm{O} 5$ & $104.2(5)$ & $\mathrm{O} 1-\mathrm{Y} 3-\mathrm{O}$ & $64.06(15)$ \\
\hline $\mathrm{O} 2 \mathrm{i}-\mathrm{Y} 2-\mathrm{O} 3$ & $79.8(2)$ & $\mathrm{O} 15-\mathrm{Y} 3-\mathrm{O}$ & $126.5(2)$ \\
\hline $\mathrm{O} 2^{\mathrm{i}}-\mathrm{Y} 2-\mathrm{O} 4^{\mathrm{i}}$ & $78.0(2)$ & $\mathrm{O} 16-\mathrm{Y} 3-\mathrm{O}$ & $130.0(3)$ \\
\hline $\mathrm{O} 3-\mathrm{Y} 2-\mathrm{O} 4^{\mathrm{i}}$ & $128.4(2)$ & $\mathrm{O} 13-\mathrm{Y} 3-\mathrm{O}$ & $126.1(2)$ \\
\hline $\mathrm{O} 2 \mathrm{i}-\mathrm{Y} 2-\mathrm{O} 1$ & $128.9(2)$ & $\mathrm{O} 14-\mathrm{Y} 3-\mathrm{O}$ & $127.7(3)$ \\
\hline $\mathrm{O} 3-\mathrm{Y} 2-\mathrm{O} 1$ & $79.2(2)$ & $\mathrm{Y} 1 \mathrm{i}-\mathrm{O}-\mathrm{Y} 1$ & 180.0 \\
\hline $\mathrm{O} 4 \mathrm{i}-\mathrm{Y} 2-\mathrm{O} 1$ & $79.7(2)$ & $\mathrm{Y} 1^{\mathrm{i}}-\mathrm{O}-\mathrm{Y} 2^{\mathrm{i}}$ & $90.07(3)$ \\
\hline $\mathrm{O} 2-\mathrm{Y} 2-\mathrm{O} 10$ & $140.8(3)$ & $\mathrm{Y} 1-\mathrm{O}-\mathrm{Y} 2^{\mathrm{i}}$ & $89.93(3)$ \\
\hline $\mathrm{O} 3-\mathrm{Y} 2-\mathrm{O} 10$ & $76.2(3)$ & $\mathrm{Y} 1-\mathrm{O}-\mathrm{Y} 2$ & 89.93 (3) \\
\hline $\mathrm{O} 4-\mathrm{Y} 2-\mathrm{O} 10$ & $140.9(3)$ & $\mathrm{Y} 1-\mathrm{O}-\mathrm{Y} 2$ & 90.07 (3) \\
\hline $\mathrm{O} 1-\mathrm{Y} 2-\mathrm{O} 10$ & $76.1(3)$ & $\mathrm{Y} 2 \mathrm{i}-\mathrm{O}-\mathrm{Y} 2$ & 180.0 \\
\hline $\mathrm{O} 2 \mathrm{i}-\mathrm{Y} 2-\mathrm{O} 11$ & $75.9(3)$ & $\mathrm{Y} 1^{\mathrm{i}}-\mathrm{O}-\mathrm{Y} 3^{\mathrm{i}}$ & $89.73(3)$ \\
\hline $\mathrm{O} 3-\mathrm{Y} 2-\mathrm{O} 11$ & $77.0(3)$ & $\mathrm{Y} 1-\mathrm{O}-\mathrm{Y} 3^{\mathrm{i}}$ & $90.27(3)$ \\
\hline $\mathrm{O} 4-\mathrm{Y} 2-\mathrm{O} 11$ & $138.8(3)$ & $\mathrm{Y} 2^{\mathrm{i}}-\mathrm{O}-\mathrm{Y} 3^{\mathrm{i}}$ & $89.76(3)$ \\
\hline $\mathrm{O} 1-\mathrm{Y} 2-\mathrm{O} 11$ & $141.2(3)$ & $\mathrm{Y} 2-\mathrm{O}-\mathrm{Y} 3^{\mathrm{i}}$ & $90.24(3)$ \\
\hline $\mathrm{O} 10-\mathrm{Y} 2-\mathrm{O} 11$ & $68.8(3)$ & $\mathrm{Y} 1-\mathrm{O}-\mathrm{Y} 3$ & $90.27(3)$ \\
\hline $\mathrm{O} 2 \mathrm{i}-\mathrm{Y} 2-\mathrm{O} 9$ & $139.4(3)$ & $\mathrm{Y} 1-\mathrm{O}-\mathrm{Y} 3$ & $89.73(3)$ \\
\hline $\mathrm{O} 3-\mathrm{Y} 2-\mathrm{O} 9$ & $140.6(3)$ & $\mathrm{Y} 2 \mathrm{i}-\mathrm{O}-\mathrm{Y} 3$ & $90.24(3)$ \\
\hline
\end{tabular}




\begin{tabular}{|c|c|c|c|}
\hline $\mathrm{O} 4 \mathrm{i}-\mathrm{Y} 2-\mathrm{O} 9$ & $76.0(3)$ & $\mathrm{Y} 2-\mathrm{O}-\mathrm{Y} 3$ & $89.76(3)$ \\
\hline $\mathrm{O} 1-\mathrm{Y} 2-\mathrm{O} 9$ & $75.8(3)$ & $\mathrm{Y} 3-\mathrm{O}-\mathrm{Y} 3$ & 180.0 \\
\hline $\mathrm{O} 10-\mathrm{Y} 2-\mathrm{O} 9$ & $68.6(3)$ & $\mathrm{Y} 1-\mathrm{O} 1-\mathrm{Y} 2$ & $97.2(2)$ \\
\hline $\mathrm{O} 11-\mathrm{Y} 2-\mathrm{O} 9$ & $105.1(4)$ & $\mathrm{Y} 1-\mathrm{O} 1-\mathrm{Y} 3$ & $97.4(2)$ \\
\hline $\mathrm{O} 2 \mathrm{i}-\mathrm{Y} 2-\mathrm{O} 12$ & $78.0(3)$ & $\mathrm{Y} 2-\mathrm{O} 1-\mathrm{Y} 3$ & $98.4(2)$ \\
\hline $\mathrm{O} 3-\mathrm{Y} 2-\mathrm{O} 12$ & $140.8(3)$ & $\mathrm{Y} 1-\mathrm{O} 2-\mathrm{Y} 3$ & $98.3(2)$ \\
\hline $\mathrm{O} 4 \mathrm{i}-\mathrm{Y} 2-\mathrm{O} 12$ & $77.3(3)$ & $\mathrm{Y} 1-\mathrm{O} 2-\mathrm{Y} 2^{\mathrm{i}}$ & $97.4(2)$ \\
\hline $\mathrm{O} 1-\mathrm{Y} 2-\mathrm{O} 12$ & $139.3(3)$ & $\mathrm{Y} 3-\mathrm{O} 2-\mathrm{Y} 2^{\mathrm{i}}$ & $99.9(2)$ \\
\hline $\mathrm{O} 10-\mathrm{Y} 2-\mathrm{O} 12$ & $102.2(3)$ & $\mathrm{Y} 1{ }^{\mathrm{i}}-\mathrm{O} 3-\mathrm{Y} 2$ & $97.3(2)$ \\
\hline $\mathrm{O} 11-\mathrm{Y} 2-\mathrm{O} 12$ & $66.5(3)$ & $\mathrm{Y} 11^{\mathrm{i}-\mathrm{O} 3-\mathrm{Y} 3}$ & $98.5(2)$ \\
\hline $\mathrm{O} 9-\mathrm{Y} 2-\mathrm{O} 12$ & $66.3(3)$ & $\mathrm{Y} 2-\mathrm{O} 3-\mathrm{Y} 3$ & $99.0(2)$ \\
\hline $\mathrm{O} 2^{\mathrm{i}}-\mathrm{Y} 2-\mathrm{O}$ & $64.02(15)$ & $\mathrm{Y} 1^{\mathrm{i}}-\mathrm{O} 4-\mathrm{Y} 2^{\mathrm{i}}$ & $97.2(2)$ \\
\hline $\mathrm{O} 3-\mathrm{Y} 2-\mathrm{O}$ & $64.31(16)$ & $\mathrm{Y} 1-\mathrm{i} 4-\mathrm{Y} 3$ & $98.3(2)$ \\
\hline $\mathrm{O} 4-\mathrm{Y} 2-\mathrm{O}$ & $64.12(16)$ & $\mathrm{Y} 2 \mathrm{i}-\mathrm{O} 4-\mathrm{Y} 3$ & $99.4(2)$ \\
\hline $\mathrm{O} 1-\mathrm{Y} 2-\mathrm{O}$ & $64.86(16)$ & & \\
\hline
\end{tabular}

Symmetry code: (i) $-x+1,-y+2,-z+2$.

Hydrogen-bond geometry (A)

\begin{tabular}{ll}
\hline$D-\mathrm{H} \cdots A$ & $D \cdots A$ \\
\hline $\mathrm{O} 7 \cdots \mathrm{O} W 2$ & $2.646(4)$ \\
$\mathrm{O} 10 \cdots \mathrm{O} W 3$ & $2.764(1)$ \\
$\mathrm{O} 13 \cdots \mathrm{O} W 4$ & $2.803(8)$ \\
$\mathrm{O} 15 \cdots \mathrm{O} W 1$ & $2.767(2)$ \\
$\mathrm{O} 16 \cdots \mathrm{O} W 4$ & $2.836(2)$ \\
$\mathrm{O} 16 \cdots \mathrm{O} W 1$ & $2.851(6)$ \\
\hline
\end{tabular}

